\title{
Refractive index sensing setup based on a taper and an intrinsic micro Fabry-Perot interferometer
}

\section{Cano-Contreras}

\section{A. D. Guzman-Chavez ad.guzman@ugto.mx \\ E. Vargas-Rodriguez \\ E. Gallegos-Arellano}

\section{Jauregui-Vazquez}

\section{R. I. Mata-Chavez \\ M. Torres-Cisneros}

\section{R. Rojas-Laguna}

\begin{abstract}
Departamento de Estudios Multidisciplinarios, División de Ingenierías, Universidad de Guanajuato, Av. Universidad s/n, Col. Yacatitas, Yuriria, Gto., C.P. 38940, Mexico Universidad Tecnológica del Suroeste de Guanajuato, Carr. Valle-Huanímaro Km. 1.2, Valle de Santiago, Gto., C.P. 38940, Mexico

Departamento de Estudios Multidisciplinarios, División de Ingenierías, Universidad de Guanajuato, Av. Universidad s/n, Col. Yacatitas, Yuriria, Gto., C.P. 38940, Mexico

Departamento de Estudios Multidisciplinarios, División de Ingenierías, Universidad de Guanajuato, Av. Universidad s/n, Col. Yacatitas, Yuriria, Gto., C.P. 38940, Mexico

Departamento de Estudios Multidisciplinarios, División de Ingenierías, Universidad de Guanajuato, Av. Universidad s/n, Col. Yacatitas, Yuriria, Gto., C.P. 38940, Mexico

Universidad Tecnológica de Salamanca, Av. Universidad Tecnológica \#200, Col. Ciudad Bajío, Salamanca, Gto., C.P. 36764, Mexico

Departamento de Electrónica, División de Ingenierías, Universidad de Guanajuato, Carretera Salamanca-Valle de Santiago Km 3.5 + 1.8, Comunidad de Palo Blanco, Salamanca, Gto., C.P. 36885, Mexico

Departamento de Estudios Multidisciplinarios, División de Ingenierías, Universidad de Guanajuato, Av. Universidad s/n, Col. Yacatitas, Yuriria, Gto., C.P. 38940, Mexico

Departamento de Electrónica, División de Ingenierías, Universidad de Guanajuato, Carretera Salamanca-Valle de Santiago Km 3.5 + 1.8, Comunidad de Palo Blanco, Salamanca, Gto., C.P. 36885, Mexico

Departamento de Electrónica, División de Ingenierías, Universidad de Guanajuato, Carretera Salamanca-Valle de Santiago Km 3.5 + 1.8, Comunidad de Palo Blanco, Salamanca, Gto., C.P. 36885, Mexico
\end{abstract}

In this work, a refractive index sensor setup based on a biconically tapered fiber (BTF) concatenated to an intrinsic all-fiber micro FabryPerot interferometer (MFPI) is presented. Here, the power of the MFPI spectral fringes decreases as the refractive index interacts with the evanescent field of the BTF segment. Furthermore, it is demonstrated that the RI sensitivity can be enhanced by bending the BTF segment. Finally, it is shown that by using this sensing arrangement, at $\sim 1.53 \mu \mathrm{m}$ wavelength, it is possible to detect refractive index changes within the measurement range of 1.3 to $1.7 \mathrm{RIU}$, with a sensitivity of $39.92 \mathrm{~dB} / \mathrm{RIU}$ and a RI resolution of $2.5 \cdot 10^{-3} \mathrm{RIU}$ with a curvature of $\mathrm{C}=18.02 \mathrm{~m}^{-1}$.

[DOI: http://dx.doi.org/10.2971/jeos.2015.15039]

Keywords: Refractive index, evanescent field, tapered fiber, Fabry-Perot interferometer, optical sensor

\section{INTRODUCTION}

The measurement of refractive index (RI) is important for a wide variety of applications, for example, in chemical and biotechnological industries, in medicine and environmental applications, etc. [1]-[4]. For many decades, RI measurement was carried out by using a refractometer [5], which usually is a bulky prisms system. In recent years, new techniques have been developed to measure RI, such as fiber optic sensors. This type of sensors have received significant attention for their unique advantages such as immunity to electromagnetic interference, small size, and capability for in-situ, and real time applications. Recently, a variety of fiber optic sensors to measure RI have been reported, some of these sensors are based on: fiber Bragg grating structures [6,7], long period gratings [8, 9], cladding mode resonance $[4,8],[10]-[12]$ and
MFPIs [8], [13]-[15]. From these, the MFPIs and the BTF are very popular options since these can be fabricated in different ways.

In this work a refractive index sensing setup based on a biconically tapered fiber and an intrinsic micro Fabry-Perot interferometer is presented. Here, the MFPI generates a well defined FPI spectrum, which interference pattern power characteristics will depend on the refractive index of the medium around the BTF concatenated to the MFPI. This change on the spectral fringe pattern is due to the interaction between the evanescent field of the BTF and the external RI. Moreover, the sensitivity of the sensing setup can be enhanced by bending the BTF, which increment the evanescent field in the cladding 


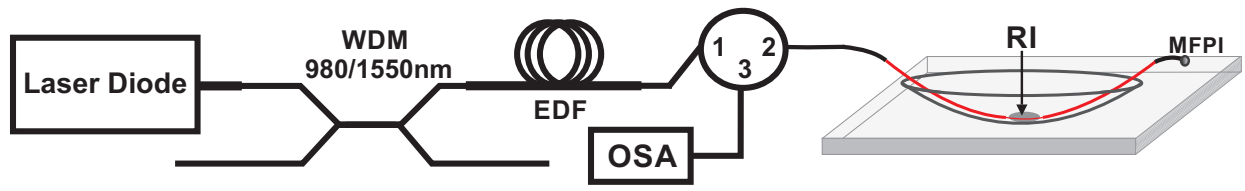

FIG. 1 Refractive index sensing setup.
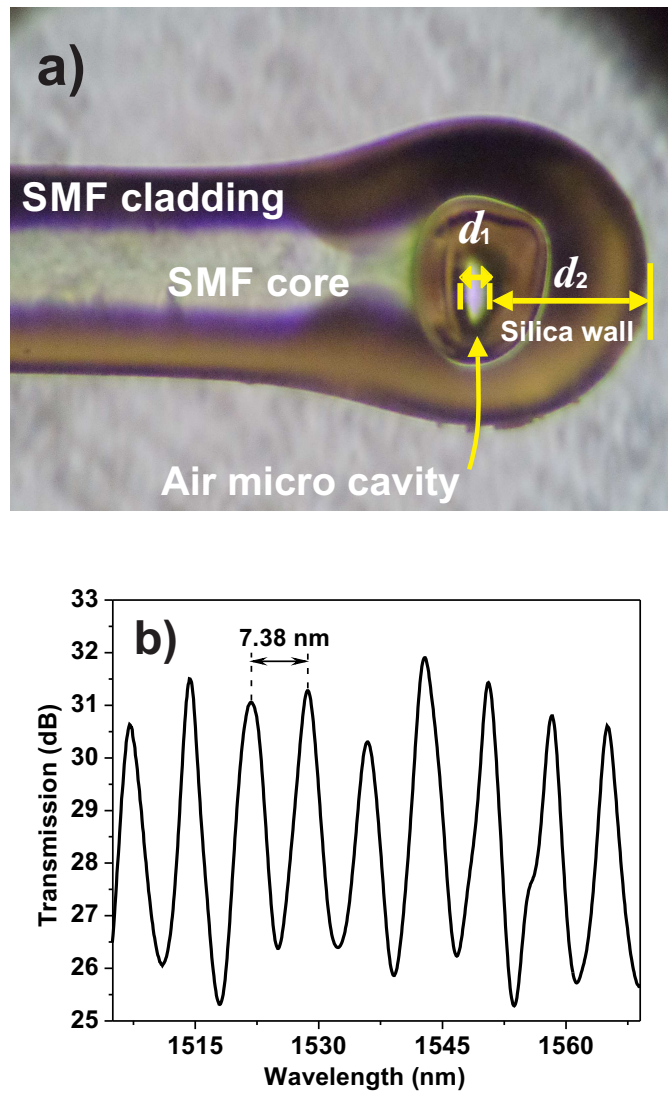

FIG. 2 (a) Picture of the MFPI and (b) the MFPI spectral response.

zone. Furthermore, the sensing element can be used for in situ applications and in general the sensing arrangement is flexible since give us the opportunity to implement a quasi real time sensor. This is because the overall sensor speed response will be mainly limited by the characteristics of the optical detector used in the setup. For the spectral range considered in our design, several photodetectors with fast response and high detectivity can be found commercially available. Finally, experimental RI measurements, within the range from 1.3 to 1.7 RIU at different curvatures, are provided.

\section{EXPERIMENTAL SETUP}

The experimental setup used to carry out the refractive index measurements is shown in Figure 1. Here, the light of a pigtailed diode laser, emitting at $\lambda=980 \mathrm{~nm}$ and delivering a maximum output power of $200 \mathrm{~mW}$, was coupled to a wavelength division multiplexer (Thorlabs BL976-SAG300) to pump an erbium doped fiber (Newport F-EDF-T3) of $3.4 \mathrm{~m}$ length. Afterwards, the luminescence generated by the EDF travels towards the sensing device (BTF-SMF-MFPI) through the circulator (Thorlabs 6015-3), from the port 1 to the port 2. Finally, the reflected interference spectrum of the MFPI was monitored at the port 3 of the circulator by using an op- tical spectrum analyzer (OSA, Yokogawa AQ6370C), which was set with a resolution of $0.02 \mathrm{~nm}$. In this arrangement the MFPI, which is based on an air microcavity, was fabricated by splicing a segment of hollow core photonic crystal fiber (HC-1064-19 Cells Fiber Crystal) to a standard single mode fiber (SMF). Here to splice both fibers a conventional arc fusion splicer (Fitel-S175) was used and the fabrication process described in detail by [16] was followed. In this process after fibres get spliced multiple electric discharges are applied in order to cleave the photonic crystal fiber. After this step an air microcavity FPI, at the tip of the SMF, is obtained. A picture of the fabricated MFPI is shown in Figure 2(a). In order to measure refractive index changes the BTF was let laid over a semi spherical cuvette and the liquids with calibrated RI were put over the BTF segment (Figure 1). Moreover, the semi spherical cuvette allowed us to apply curvature to the BTF segment.

\subsection{Operating principle of the sensing device}

The principle of operation of our sensing device is explained by means of the interference phenomenon occurring within the MFPI and by the losses due to the RI surrounding the BTF segment. The interference spectrum of the MFPI used in this work is formed mainly by two spectral fringe patterns occurring one at the air cavity, with length $d_{1}$, and the other one occurring at the exit thin silica wall of the MFPI, with length $d_{2}$ (Figure 2(a)). The length of these optical cavities $\left(d_{1}\right.$ and $\left.d_{2}\right)$ can be varied by changing the distance between the SMF and the PCF when these are spliced, by changing the point where the electrical discharges are applied to cleave the fiber to form the MFPI and by the number of arc discharges applied [16]. Hence, in Figure 2(b) the measured transmittance pattern of our MFPI is shown, where it can be observed that the main well defined FPI spectrum has a $\Delta \lambda=7.38 \mathrm{~nm}$. For a single cavity FPI the separation between two consecutive spectral fringes it is known as the free spectral range $(\Delta \lambda)$ and it can be calculated by using the formula:

$$
\Delta \lambda=\frac{\lambda^{2}}{2 n d}
$$

where $\lambda$ is the reference wavelength and $n d$ is the optical thickness of the FPI cavity, being for our case $n_{2}=1.44$. Therefore by using Eq. (1) it is possible to determine that the length (thickness) of the exit thin silica wall is around $d_{2}=110.9 \mu \mathrm{m}$ (Figure 2(a)). The air microcavity has a length $d_{1}=12.7 \mu \mathrm{m}$, it was measured by using the fusion splicer camera. Afterwards, the reflected interference spectrum of the MFPI will pass through the BTF segment. The waist length and diameter of this BTF segment were $2.5 \mathrm{~cm}$ and $70 \mu \mathrm{m}$ respectively. Here, let us to recall that when the RI surrounding the BTF segment is varied, losses in the guided light transmission will occur, since the RI interacts with the evanescent field of the cladding zone [17]. In a tapered fiber the evanescent field at the external 


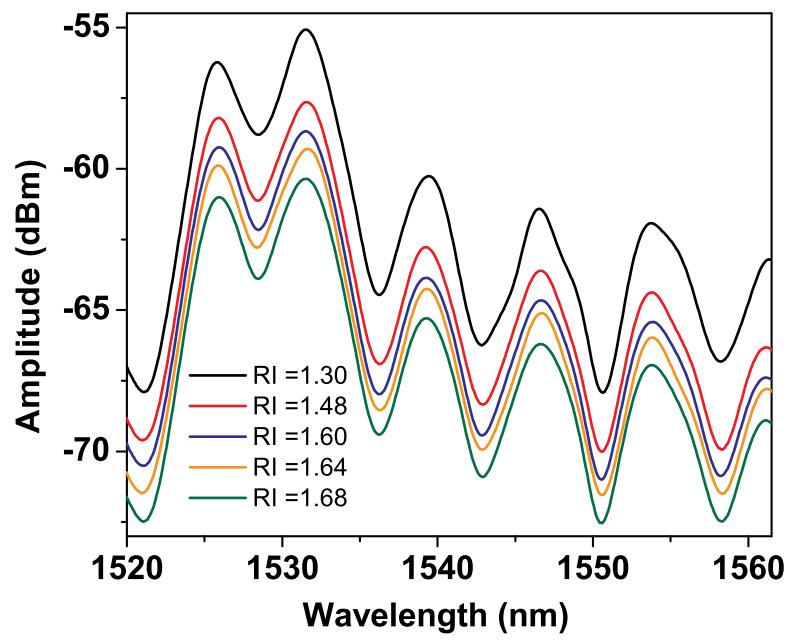

FIG. 3 Measured MFPI spectra for different refractive index values and with curvature $C=0 \mathrm{~m}^{-1}$

medium is increased since a greater portion of light is guided by the cladding [18], which is due to fact that by the tapering fiber process the cladding and the core are reduced in the same proportion. In this way changes on the refractive index of the external medium will induce a variation of the effective index of propagation in a tapered fiber optic, thus changing the confinement conditions of transmitted light by the tapered fiber. Moreover, as the external refractive index increases therefore it is expected that the guided light gets attenuated due to the effects over the total internal reflection characteristics, which consequently means that a portion of light will not be coupled back into the core fiber after the BTF segment.

Furthermore, when the BTF segment is bent with a certain curvature radius, the field distribution is modified $[19,20]$, producing that some light travelling by the core leaks into the cladding increasing the evanescent field. Therefore, the refractive index sensitivity can be enhanced by increasing the interaction between the evanescent field of the BTF and the external RI.

\section{EXPERIMENTAL RESULTS}

Reflected spectra for different refractive index values with curvature $C=0 \mathrm{~m}^{-1}$ and generated using a pump power of $100 \mathrm{~mW}$ are shown in Figure 3. Here it can be observed that as the refractive index increases, the reflected power is attenuated. This is because of the interaction between the external RI and the evanescent field in the BTF segment, as was previously mentioned. It is important to comment that to carry out our experiments, liquids with calibrated refractive index and transparent at $\lambda \sim 1.5 \mu \mathrm{m}$ (Cargille labs) were used. Moreover, from Figure 3 can be seen that the sensitivity of each fringe depends on the shape of the source spectrum, in our case the luminescence spectrum of the erbium. For instance, the fringe centered at $\lambda \sim 1.53 \mu \mathrm{m}$ has a greater sensitivity than the one centered at $\lambda \sim 1.55 \mu \mathrm{m}$.

The reflected power of two fringes as a function of the external RI, at two different curvatures is presented in Figure 4. Here it can be observed that exist a significant relationship

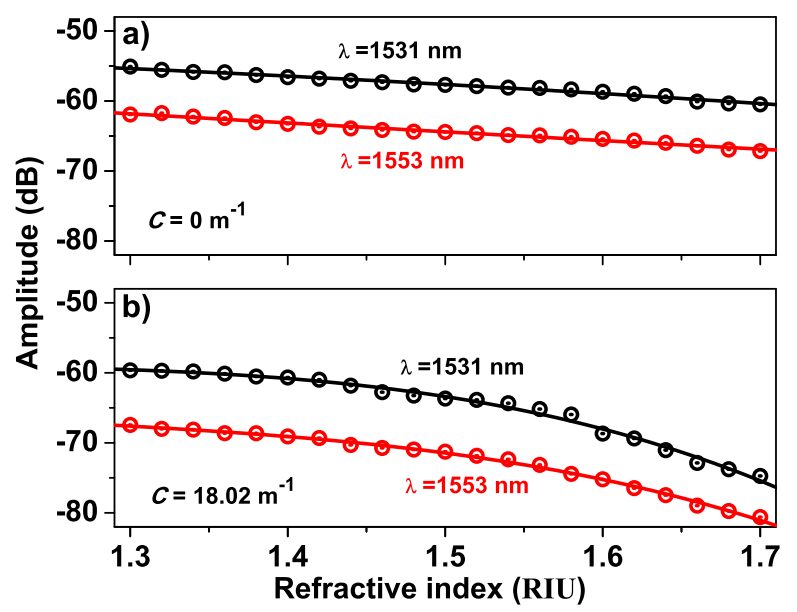

FIG. 4 Measured of the reflected power for two MFPI spectral fringes as a function of the external refractive indexes with curvatures: (a) $C=0 \mathrm{~m}^{-1}$ and (b) $C=18.02 \mathrm{~m}^{-1}$.

between the intensity of the FPI fringes and the external refractive index. Also it can be noted that the RI sensitivity is enhanced when the BTF is bent. For the case when the curvature of the BTF segment was $C=0 \mathrm{~m}^{-1}$ the RI sensitivity for the fringes centered at $\lambda \sim 1.53 \mu \mathrm{m}$ and $\lambda \sim 1.55 \mu \mathrm{m}$ were $12.78 \mathrm{~dB} / \mathrm{RIU}$ and $12.35 \mathrm{~dB} / \mathrm{RIU}$ respectively, within the range from 1.3 to 1.7 RIU. Moreover, when the curvature of the BFT segment was $C=18.02 \mathrm{~m}^{-1}$ the maximum RI sensitivity for the fringes centered at $\lambda \sim 1.53 \mu \mathrm{m}$ and $\lambda \sim 1.55 \mu \mathrm{m}$ were $39.92 \mathrm{~dB} /$ RIU and $33.35 \mathrm{~dB} /$ RIU respectively within the same RI range. Comparing these values, it can be appreciated how the bending helps to enhance the overall sensor performance. Moreover, by considering that the OSA used to perform the experiments has an amplitude resolution of $0.1 \mathrm{~dB}$, it is possible to state that the curvature resolution for this arrangement is in the order of $2.5 \cdot 10^{-3} \mathrm{~m}^{-1}$, this for the fringe centered at $\lambda \sim 1.53 \mu \mathrm{m}$ with a curvature of $C=18.02 \mathrm{~m}^{-1}$ on the BTF segment. Finally, in our case the effects due to temperature over the sensing element can be considered as negligible since the thermo optic coefficient of silica is very small $\left(+0.5 \cdot 10^{-6}{ }^{\circ} \mathrm{C}^{-1}\right)[21]$.

It is important to point out that measurements reported in this work were taken directly with the OSA, however for practical reasons it must be replaced, ideally, by a simple optical stage and low cost photodetectors. Here the spectrum generated by the FPI concatenated with the ATF (Figure 3) give us the opportunity to implement different simple sensor configurations. For instance if we would like to consider only the reflected power at certain wavelength (Figure 4) it can be useful to include a fiber optical coupler, before the port 1 of the circulator, to split the light source beam in order to have one reference channel. The port 3 of the circulator will provide the measurement channel. In this way two optical detectors can measure the exit power of each channel, afterwards these measurements can be correlated to obtain the deep of modulation, which helps to compensate drifting errors, that are very usual in this type of sensing arrangements, due to power source variations [22]. Another option in which the FPI spectral fringe pattern can be very useful, is to implement a cross correlation setup. This can be achieved by adding to our arrangement an extra simple interferometer, which is 
necessary to produce a second modulated spectrum. Hence to implement this kind of sensor it is possible to pass the output beam, provided by the port 3 of the circulator, by another interferometer which will superimpose another modulated spectrum that will serve as a reference. In this way the modulated spectrum produced by the micro FPI will be affected by changes occurring at the measurand, while the reference spectrum will serve to cross correlate both spectra. This type of sensors have the advantage to be very sensitive only to changes occurring in the measurand and also are very robust to drifting errors [23]-[25].

\section{CONCLUSIONS}

In this work a refractive index sensor setup based on a biconically tapered fiber (BTF) concatenated to a fiber micro FabryPerot interferometer was demonstrated. Here, the reflected power of the interference pattern, generated by the MFPI, was modified by the interaction between the refractive index with the evanescent field of the tapered fiber. It was shown that the refractive index sensitivity can be enhanced when the tapered fiber is bent taking advantage of the increment of the evanescent field on the cladding zone. In this way when the BTF segment was bent with a curvature of $C=18.02 \mathrm{~m}^{-1}$, the sensitivity and the resolution of our sensing arrangement were $39.92 \mathrm{~dB} / \mathrm{RIU}$ and $2.5 \cdot 10^{-3}$ RIU respectively, within the refractive index range from 1.3 to 1.7 RIU. Finally, it is important to mention that this kind of sensor can be enhanced by adding a simple optical stage and low cost photodetectors which can help to increase the sensitivity and to reduce drifting errors.

\section{ACKN OWLEDGEMENTS}

This work was developed with support in part from CONCYTEG under projects 14-PNPC-DPP-Q182-47, 14-PNPCDPP-Q182-49 and 14-IJ-DPP-Q182-46, in part from Catedras Patrimoniales de la UGTO and in part by SEP-PRODEP under project UGTO-PTC-341 and PIFI2015.

\section{References}

[1] A. Leung, P. M. Shankar, and R. Mutharasan, "A review of fiberoptic biosensors," Sensor Actuat. B-Chem. 125, 688-703 (2007).

[2] M. Mehrvar, C. Bis, J. M. Scharer, M. M. Young, and J. H. Luong, "Fiber-optic biosensors-trends and advances," Anal. Sci. 16, 677-692 (2000).

[3] M. Marazuela, and M. Moreno-Bondi, "Fiber-optic biosensors - an overview," Anal. Bioanal Chem. 372, 664-682 (2002).

[4] T. K. Yadav, R. Narayanaswamy, M. H. Abu Bakar, Y. M. Kamil, and M. A. Mahdi, "Single mode tapered fiber-optic interferometer based refractive index sensor and its application to protein sensing," Opt. Express 22, 22802-22807 (2014).

[5] M. Born, and E. Wolf, Principles of optics (Great Britain: Pergamon Press, Oxford, 1970).
[6] A. P. Zhang, G. Yan, S. Gao, S. He, B. Kim, J. Im, and Y. Chung, “Microfluidic refractive-index sensors based on small-hole microstructured optical fiber Bragg gratings," Appl. Phys. Lett. 98, 221109 (2011).

[7] W. Liang, Y. Huang, Y. Xu, R. K. Lee, and A. Yariv, “Highly sensitive fiber Bragg grating refractive index sensors," Appl. Phys. Lett. 86, 151122 (2005).

[8] P. A. S. Jorge, S. O. Silva, C. Gouveia, P. Tafulo, L. Coelho, P. Caldas, D. Viegas, et al., "Fiber optic-based refractive index sensing at INESC Porto," Sensors 12, 8371-8389 (2012).

[9] A. Lim, W. B. Ji, and S. C. Tjin, "Improved refractive index sensitivity utilizing long-period gratings with periodic corrugations on cladding," J. Sensor 2012, 48347 (2012).

[10] R. Yang, Y.-S. Yu, Y. Xue, C. Chen, Q.-D. Chen, and H.-B. Sun, "Single S-tapered fiber Mach-Zehnder interferometers," Opt. Lett. 36, 4482-4484 (2011).

[11] L.-P. Sun, J. Li, Y. Tan, S. Gao, L. Jin, and B.-0. Guan, "Bending effect on modal interference in a fiber taper and sensitivity enhancement for refractive index measurement," Opt. Express 21, 26714-26720 (2013).

[12] L. Xu, Y. Li, and B. Li, "Nonadiabatic fiber taper-based MachZehnder interferometer for refractive index sensing," Appl. Phys. Lett. 101, 153510 (2012).

[13] 0. Frazão, P. Caldas, J. L. Santos, P. V. S. Marques, C. Turck, D. J. Lougnot, and 0. Soppera, "Fabry-Perot refractometer based on an end-of-fiber polymer tip," Opt. Lett. 34, 2474-2476 (2009).

[14] 0. Frazão, J. M. Baptista, J. L. Santos, J. Kobelke, and K. Schuster, "Refractive index tip sensor based on Fabry-Perot cavities formed by a suspended core fibre," J. Eur. Opt. Soc.- Rapid 4, 09041 (2009).

[15] M. Deng, C.-P. Tang, T. Zhu, Y.-J. Rao, L.-C. Xu, and M. Han, “Refractive index measurement using photonic crystal fiber-based FabryPerot interferometer," Appl. Opt. 49, 1593-1598 (2010).

[16] D. Jauregui-Vazquez, J. M. Estudillo-Ayala, R. Rojas-Laguna, E. Vargas-Rodriguez, J. M. Sierra-Hernandez, J. C. HernandezCarcia, and R. I. Mata-Chavez, "An all fiber intrinsic Fabry-Perot interferometer based on an air-microcavity," Sensors 13, 6355-6364 (2013).

[17] E. Baude, "Numerical study of tapered fiber optics as evanescent field sensors," in Proceedings to 2013 SBMO/IEEE MTT-S International Microwave O Optoelectronics Conference (IMOC), 1-5 (IEEE, Rio de Janeiro, 2013).

[18] A. Hartung, F. Wirth, and H. Bartelt, "Light propagation in tapered optical fibers: Spatial light confinement and generation of plasmonic waves,", in Proceedings to Progress in Electromagnetics Research Symposium, 255-258 (The Electromagnetics Academy, Marrakesh, 2011).

[19] L. C. Bobb, P. M. Shankar, and H. D. Krumboltz, "Bending effects in biconically tapered single-mode fibers," J. Lightwave Technol. 8, 1084-1090 (1990).

[20] D. Marcuse, "Curvature loss formula for optical fibers," J. Opt. Soc. Am. 66, 216-220 (1976).

[21] T. Zhu, D. Wu, M. Liu, and D.-W. Duan, "In-line fiber optic interferometric sensors in single-mode fibers," Sensors 12, 10430-10449 (2012).

[22] P. Chambers, E. A. D. Austin, and J. P. Dakin, "Theoretical analysis of a methane gas detection system, using the complementary source modulation method of correlation spectroscopy," Mea. Sci. Technol. 15, 1629 (2004). 
[23] R. Goody, "Cross-correlating spectrometer," J. Opt. Soc. Am. B 58, 900-908 (1968).

[24] E. Vargas-Rodriguez, and H. N. Rutt, "Method to minimize spurious background signals in gas detectors based on correlation spectroscopy using a Fabry-Perot bandpass filter shape optimization," Opt. Eng. 44, 103002 (2005).
[25] E. Vargas-Rodríguez, and H. N. Rutt, "An analytical method to find the optimal parameters for gas detectors based on correlation spectroscopy using a Fabry-Perot interferometer," Appl. Optics 46, 4625-4632 (2007). 